\title{
Remarkable Size Effect on Photophysical and Nonlinear Optical Properties of All-Carboatomic Rings, Cyclo[18]carbon and Its Analogues
}

\author{
Zeyu Liu ${ }^{\mathrm{a}, *}$, Tian Lu ${ }^{\mathrm{b}, *}$, Aihua Yuan ${ }^{\mathrm{a}}$, Qinxue Chen ${ }^{\mathrm{b}}$, Xiufen Yan ${ }^{\mathrm{a}}$ \\ ${ }^{a}$ School of Environmental and Chemical Engineering, Jiangsu University of Science and \\ Technology, Zhenjiang 212100, People's Republic of China \\ ${ }^{b}$ Beijing Kein Research Center for Natural Sciences, Beijing 100022, People's Republic of \\ China
}

${ }^{*}$ Corresponding author. E-mail: liuzy@ just.edu.cn (Zeyu Liu)

${ }^{*}$ Corresponding author. E-mail: sobereva@ sina.com (Tian Lu) 


\begin{abstract}
Inspired by recent experimental observation of molecular morphology and theoretical predictions of multiple properties of cyclo[18]carbon, we systematically studied photophysical and nonlinear optical properties of cyclo[2N]carbons $(N=3-15)$ allotropes through density functional theory. This work unveils the unusual optical properties of the sp-hybridized carbon rings with different sizes. The remarkable size dependence of optical properties of these systems and its underlying natures are profoundly explored, and the relevance between aromaticity and optical properties are highlighted. The extrapolation curves fitted for energy level of frontier molecular orbitals, maximum absorption wavelength, and (hyper)polarizability of considered carbon rings are presented, which can be used to reliably predict corresponding properties for arbitrarily large rings. The findings in this study will facilitate the exploration of potential application of the cyclocarbons in the field of optical material.
\end{abstract}

\title{
Table of contents (TOC) graphic:
}

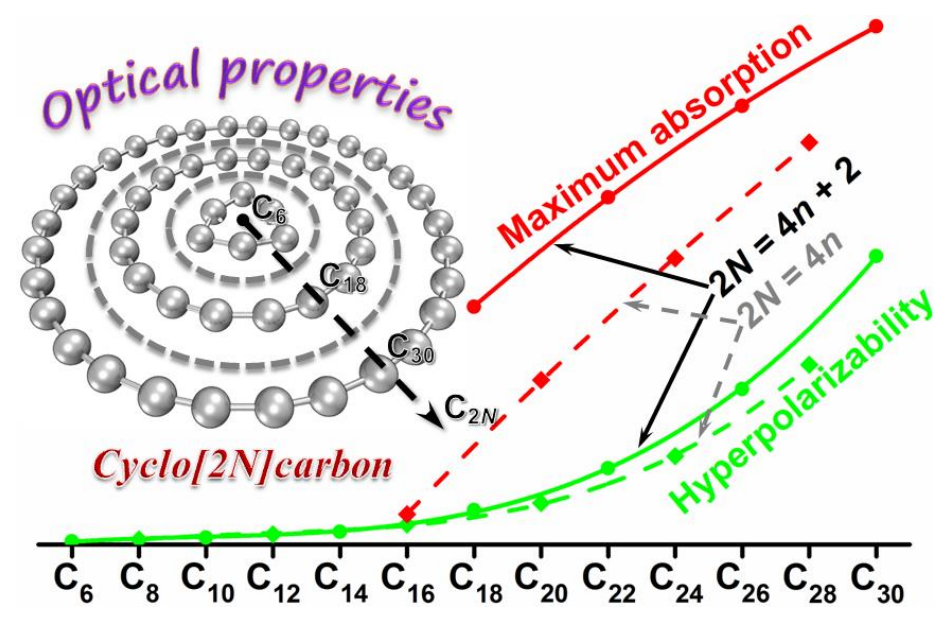




\section{Introduction}

Cyclo[18]carbon $\left(\mathrm{C}_{18}\right)$, a closed-loop polyyne of 18 sp-hybridized carbon atoms joined together, is one of the hottest molecule recently. Due to its unique molecular geometry and electronic structure, $\mathrm{C}_{18}$ has quickly attracted extensive attentions from chemists after being generated and observed in condensed phase in $2019^{1}$ and soon received wide investigations ${ }^{2-18}$. Our group has also made a series of theoretical studies on it, including aromaticity, optical properties, intermolecular interaction, vibrational spectra, and so on ${ }^{19-23}$. These works found that this novel molecule displays very distinctive characteristics from conventional chemical systems, and thus has great potential for constructing materials with unusual performances.

Very lately, a new method of preparing $\mathrm{C}_{18}$ based on debromination of $\mathrm{C}_{18} \mathrm{Br}_{6}$ has been reported ${ }^{24}$, and it was mentioned that precursors of cyclic oligomers with other sizes, such as $\mathrm{C}_{12} \mathrm{Br}_{4}$ and $\mathrm{C}_{24} \mathrm{Br}_{8}$, have also been detected in the reaction mixture. It is implied that the realization of different sizes of cyclocarbons other than $\mathrm{C}_{18}$ should be possible in the foreseeable future.

Optical properties, such as electronic absorption spectrum and (hyper)polarizability, are important molecular characteristics because they determine the possibility that molecules will eventually be used as practical optical materials. We have expounded that the $\mathrm{C}_{18}$ exhibits extraordinarily strong absorption in ultraviolet region and possesses striking optical nonlinearity ${ }^{20}$, as well, the vibrational spectra of $\mathrm{C}_{2 N}$ change regularly with the ring size and are closely related to the parity of $N^{22}$. Therefore, it is quite appealing to further study the photophysical and nonlinear properties of $\mathrm{C}_{2 N}$ allotropes with regard to the ring size. Here, we explore this topic in detail by density functional theory calculation for a series of $\mathrm{C}_{2 N}$ molecules ranging from very small size $\left(\mathrm{C}_{6}, N=3\right)$ to fairly large one $\left(\mathrm{C}_{30}, N=15\right)$ (Scheme 1$)$, and the calculated properties are further extrapolated to larger systems. All computational details are given at the end of this article. This study will not only help to shed light on the important relationship between the optical properties and the size of the investigated carbon rings, but also provide a general strategy of predicting these properties for their arbitrarily large analogues, which could be used to screen out cyclocarbons with specific properties. 


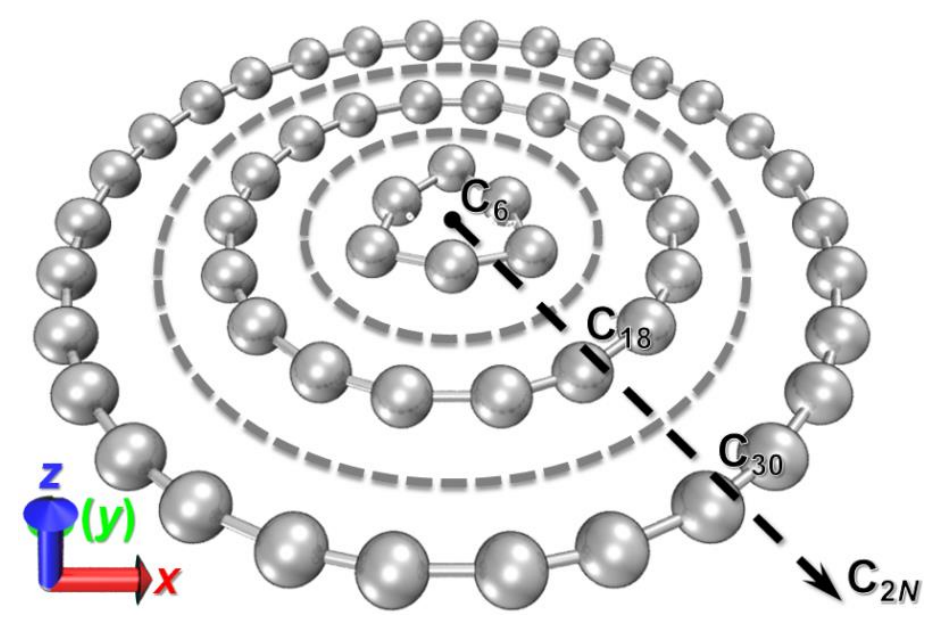

Scheme 1. Optimized geometry of three representative cyclocarbons in $\mathrm{C}_{2 N}$ allotropes studied in this work. Also shown is the Cartesian axis.

\section{Computational details}

The geometry optimizations of the $\mathrm{C}_{2 N}$ allotropes were carried out by density functional theory (DFT) using $\omega \mathrm{B} 97 \mathrm{XD}$ exchange-correlation functional ${ }^{25}$ in combination with def2-TZVP basis set ${ }^{26}$ in vacuum. Frequency analyses have been performed at the same level to confirm that there is no imaginary frequency. The excitation energies and oscillator strengths of the first 50 singlet excited states of the $\mathrm{C}_{2 N}$ calculated by time-dependent DFT (TDDFT) at the $\omega \mathrm{B} 97 \mathrm{XD} / \mathrm{def} 2-\mathrm{TZVP}$ level were used to simulate their UV-Vis absorption spectra. This computational strategy has been shown to be reliable for $\mathrm{C}_{18}{ }^{19,20}$ and thus expected to be also suitable for other kinds of all-carboatomic rings. The average atomization energy, namely the atomization energy divided by number of ring members, for different $\mathrm{C}_{2 N}$ was calculated at the accurate $\omega \mathrm{B} 97 \mathrm{M}-\mathrm{V}^{27} / \mathrm{def} 2-\mathrm{QZVPP}{ }^{26}$ level. Molecular polarizabilities $(\alpha)$ and second-order hyperpolarizabilities $(\gamma)$ were determined using analytic derivatives of electronic energy by solving coupled-perturbed Kohn-Sham equation ${ }^{28}$ at CAM-B3LYP ${ }^{29} /$ aug-cc-pVTZ(-f) level, where aug-cc-pVTZ(-f) is a reduced version of aug-cc-pVTZ basis set ${ }^{30}$ with removal of f-type polarization functions. By comparing our previous results of $\mathrm{C}_{18}{ }^{20}$, it was found that this calculation strategy can perfectly reproduce the response properties calculated under the much larger basis set $\left(\right.$ LPol-ds) ${ }^{31}$ specifically developed for accurate estimation of molecular hyperpolarizability, showing the reliability of the current calculation level.

Detailed formulae for calculating molecular (hyper)polarizabilities are described as 
follows. For the static case, Taylor expansion of total energy $E(F)$ of a system with respect to external electric field $F$ is expressed as ${ }^{32,33}$ :

$$
\begin{aligned}
E(F)= & E(0)-\sum_{i} \mu_{i} F_{i}-\frac{1}{2 !} \sum_{i} \sum_{j} \alpha_{i j} F_{i} F_{j}-\frac{1}{3 !} \sum_{i} \sum_{j} \sum_{k} \beta_{i j k} F_{i} F_{j} F_{k} \\
& -\frac{1}{4 !} \sum_{i} \sum_{j} \sum_{k} \sum_{l} \gamma_{i j k l} F_{i} F_{j} F_{k} F_{l}-\cdots \quad i, j, k, l=\{x, y, z\}
\end{aligned}
$$

where $\mu_{i}, \alpha_{i j}, \beta_{i j k}$, and $\gamma_{i j k l}$ are the tensor components of dipole moment, polarizability, first-order hyperpolarizability, and second-order hyperpolarizability, respectively. This expression corresponds to T-convention of Willetts et al. ${ }^{34}$ Formulae of some terms related to molecular response properties involved in this work are given below:

Polarizability anisotropy $\left(\alpha_{\text {aniso }}\right)$ :

$$
\alpha_{\text {aniso }}=\sqrt{\left[\left(\alpha_{x x}-\alpha_{y y}\right)^{2}+\left(\alpha_{y y}-\alpha_{z z}\right)^{2}+\left(\alpha_{z z}-\alpha_{x x}\right)^{2}+6\left(\alpha_{x y}^{2}+\alpha_{y z}^{2}+\alpha_{x z}^{2}\right) / 2\right]}
$$

Isotropic polarizability $\left(\alpha_{\text {iso }}\right)$

$$
\alpha_{\text {iso }}=\left(\alpha_{x x}+\alpha_{y y}+\alpha_{z z}\right) / 3
$$

Average second-order hyperpolarizability $\left(\gamma_{\|}\right)$

$$
\gamma_{\|}=(1 / 15) \sum_{i, j}\left(\gamma_{i j j j}+\gamma_{i j j i}+\gamma_{i j i j}\right) \quad i, j=\{x, y, z\}
$$

Meanwhile, the electron density of a system $\rho(\vec{r}, F)$ can be defined with respect to the external field $F$ :

$$
\begin{array}{r}
\rho(\vec{r}, F)=\rho^{(0)}(\vec{r})+\sum_{i} \rho_{i}^{(1)}(\vec{r}) F_{i}+\frac{1}{2 !} \sum_{i} \sum_{j} \rho_{i j}^{(2)}(\vec{r}) F_{i} F_{j}+\frac{1}{3 !} \sum_{i} \sum_{j} \sum_{k} \rho_{i j k}^{(3)}(\vec{r}) F_{i} F_{j} F_{k}+\cdots \\
i, j, k=\{x, y, z\}
\end{array}
$$

The component of polarizability is expressed by:

$$
\alpha_{i j}=\int-\vec{r}_{i} \rho_{j}^{(1)}(\vec{r}) d \vec{r} \quad i, j=\{x, y, z\}
$$

The component of second-order hyperpolarizability is expressed by:

$$
\gamma_{i j k l}=\int-\vec{r}_{i} \rho_{j k l}^{(3)}(\vec{r}) d \vec{r} \quad i, j, k, l=\{x, y, z\}
$$

Gaussian 16 (A.03) package ${ }^{35}$ was employed for all quantum chemistry calculations except for the average atomization energy, which was calculated by ORCA 4.2.1 program $^{36}$. The electronic structure analyses were performed with the 
Multiwfn 3.7 code $^{37}$. The isosurface maps of real space functions were rendered by means of Visual Molecular Dynamics (VMD) software ${ }^{38}$ based on the files exported by Multiwfn.

\section{Results and Discussion}

\subsection{Geometry, stability, and electronic structure of $C_{2 N}$ allotropes}

The optimized structures of all $\mathrm{C}_{2 N}$ molecules were found to be strictly planar by checking the resulting coordinates given in Table $\mathrm{S} 1$. The medium to large $\mathrm{C}_{2 N}$ rings $(N \geq 8)$ possess $D_{N \mathrm{~h}}$ symmetry with alternating bond lengths and constant bond angle, while the symmetry of small rings are not uniform. Although $\mathrm{C}_{6}$ and $\mathrm{C}_{10}$ are also $D_{N \mathrm{~h}}$ symmetrical, they show alternating bond angles but invariant bond length, while $\mathrm{C}_{8}$, $\mathrm{C}_{12}$, and $\mathrm{C}_{14}$ belong to lower-order point group $\left(C_{N \mathrm{~h}}\right)$ with simultaneously alternating bond lengths and angles.

The relative stability of various $\mathrm{C}_{2 N}$ allotropes is believed to have close relevance to the possibility of practical syntheses and applications and thus worth to be investigated. We defined average atomization energy, namely the atomization energy divided by the number of ring members, to appraise it (Figure S1). In general, it can be seen that the larger the size of the ring, the more stable it is, and the stability converges smoothly starting from $\mathrm{C}_{14}$. We note that especially for small rings, when $N$ changes from even $(2 N=4 n)$ to odd $(2 N=4 n+2)$, the improvement of stability is particularly significant, and $\mathrm{C}_{10}$ is even slightly more stable than $\mathrm{C}_{12}$. A possible explanation of this observation is that both in-plane and out-of-plane $\pi$ electrons $\left(\pi^{\text {in }}\right.$ and $\pi^{\text {out }}$ electrons, see Ref. 19 for details) in $\mathrm{C}_{2 N}$ systems with odd number of $N$ satisfy Hückel $(4 n+2)$ rule, which notably stablizes the systems.

We plotted the total density-of-states (TDOS) and partial density-of-states (PDOS) curves contributed by various sets of valence double-occupied molecular orbitals (MOs) for all $\mathrm{C}_{2 N}$ systems (Figure $\mathrm{S} 2$ ), including $2 N \sigma \mathrm{MOs}, N \pi^{\text {in }} \mathrm{MOs}$, and $N \pi^{\text {out }}$ MOs indexed in Table S2 (see Ref. 20 for details). They clearly show that except for the smallest ring $\mathrm{C}_{6}$, the $\sigma$ MOs of the cyclocarbons exhibit much lower energy than the $\pi$ MOs, while the energy difference between the $\pi^{\text {in }}$ and $\pi^{\text {out }}$ MOs is almost indistinguishable. The heights of the spikes in the DOS maps indicate that most MOs are doubly degenerate, which stems from the high symmetry of the $\mathrm{C}_{2 N}$. The levels of highest occupied molecular orbital (HOMO) and the lowest unoccupied molecular 
orbital (LUMO) of $\mathrm{C}_{2 N}$ from $\mathrm{C}_{16}$ are fitted with respect to atomic number according to the parity of the $N$ (Figure S3). The near-perfect fitting quality $\left(R^{2}>0.999\right)$ suggests that it can be used to predict the energy level of frontier molecular orbitals (FMOs) of larger carbon rings.

\subsection{Photophysical property of $C_{2 N}$ allotropes}

The absorption bands of all considered $\mathrm{C}_{2 N}$ molecules [Figure 1(a)] are evidently located in the ultraviolet region, so they should be colorless. With the enlargement of ring size, the absorption shifts notably towards long wavelength and the peak intensity increases obviously, displaying very regular ring-size dependence of the absorption spectrum. It is also worth to note that the cyclocarbons with even $N$ has significantly stronger absorption than its neighbours whose $N$ is odd. We fitted quadratic curves upon the maximum absorption wavelength of $\mathrm{C}_{2 N}(N \geq 8)$ with respect to the number of carbons [Figure 1(b)] and perfect fitting curves with $R^{2} \approx 1$ are obtained. The maximum absorption wavelength is found to be less than the lower limit of visible light (about $380 \mathrm{~nm}$ ) even at the apex of the quadratic curve regardless of the parity of $N$, indicating that cyclocarbon of any size is unlikely to display color.
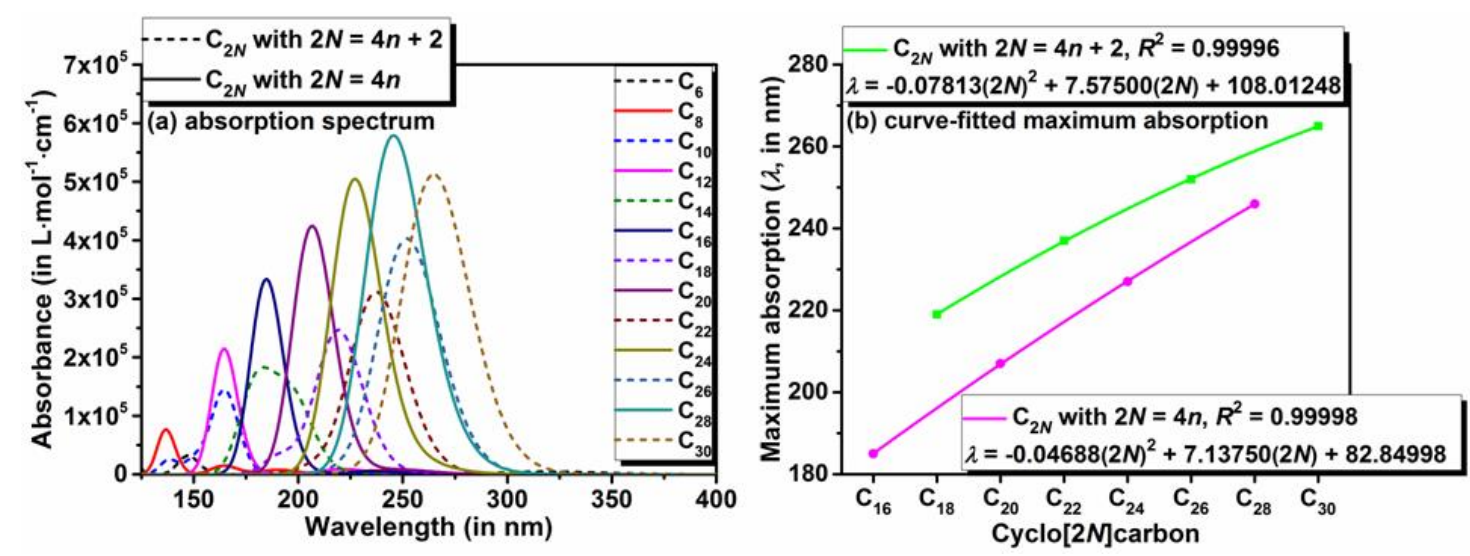

Figure 1. (a) Simulated electronic absorption spectrum of $\mathrm{C}_{2 N}$ allotropes and (b) curve-fitted maximum absorption wavelength of the $\mathrm{C}_{2 N}$ systems (from $\mathrm{C}_{16}$ ) with respect to atomic number according to the parity of the $N$. The Gaussian function with full width at half-maximum of $0.333 \mathrm{eV}$ was employed for broadening the theoretical data as spectrum curves.

The maximum absorption bands of all $\mathrm{C}_{2 N}$ systems are found to be caused by $\pi-\pi *$ transition according to the hole-electron analysis (Figure S4) ${ }^{20}$, since both hole and 
electron of corresponding electron excitation are distributed around C-C bond axes. The reason for the red-shift of maximum absorption of the $\mathrm{C}_{2 N}$ with increasing its size should be closely related to the common phenomenon that as number of repeating units of a $\pi$-conjugated oligomer increases, the energies of HOMO and LUMO rise and drop, respectively ${ }^{39}$, implying overall increase of $\pi$ occupied MOs and decrease of $\pi *$ unoccupied ones, which result in lowered $\pi-\pi *$ excitation energies. HOMOLUMO gaps and maximum absorption wavelengths of all studied cyclocarbons are collectively plotted in Figure 2, from which it can be seen that the two properties are indeed closely related to each other and share the same variation trend. However, $\mathrm{C}_{10}$ shows a distinctive difference with significantly large gap. It is notable that the aromaticity of $\mathrm{C}_{10}$ as distinct from that of other carbon rings has been reported ${ }^{18}$, and some works have revealed that molecular aromaticity can serve as a fingerprint of optical properties in organic macrocycles ${ }^{40-42}$, which jointly explain its anomaly.

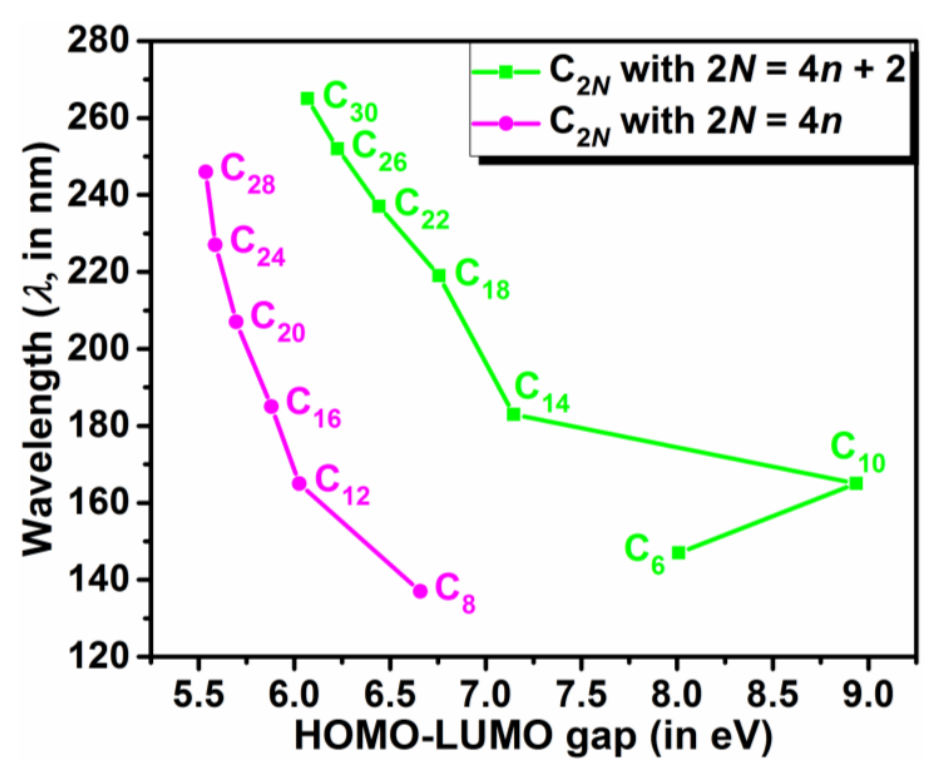

Figure 2. Plot of the HOMO-LUMO gaps versus maximum absorption wavelengths for $\mathrm{C}_{2 N}$ systems with respect to atomic number according to the parity of the $N$.

Oscillator strength $(f)$ of an excited state is proportional to the square of transition dipole moment $\left(\mu^{\mathrm{T}}\right)$ corresponding to this state, while transition dipole moment density is able to intuitively reveal the contribution of different spatial positions to $\mu^{\mathrm{T} 43}$. As clearly shown in Figure S5, the transition dipole moment density is mostly distributed at both ends of the coordinate axis, and its magnitude increases with the increase of ring size. This observation suggests that the regions at the two ends of the 
coordinate axis make main contribution to the $\mu^{\mathrm{T}}$, and larger rings tend to have larger $\mu^{\mathrm{T}}$ and thus possess greater $f$, which in turn leads to stronger absorption.

\subsection{Nonlinear optical property of $C_{2 N}$ allotropes.}

The response properties of the $\mathrm{C}_{2 N}$ systems in zero-frequency limit $(\lambda=\infty \mathrm{nm})$ and frequency-dependent fields $(\lambda=1907,1460$, and $1064 \mathrm{~nm})$ (Table S3) were calculated by CPKS method ${ }^{28}$. All carbon rings show remarkable polarizability anisotropy [ $\alpha_{\text {aniso }}(\infty)$ ] [Figure 3(a)], which is in line with the fact that the components of polarizability tensor parallel to the ring $\left[\alpha_{x x}(\infty)\right.$ and $\left.\alpha_{y y}(\infty)\right]$ are significantly larger than that perpendicular to the ring $\left[\alpha_{z z}(\infty)\right]$. Undoubtedly, this results from the extensive delocalization of the $\pi$ electrons over the cyclocarbons, which makes the charge distribution of these system have a very strong response to the electric field parallel to the ring plane. In order to more intuitively exhibit the interesting character of the response property, the unit sphere representation map ${ }^{44}$ of polarizability tensor of $\mathrm{C}_{18}$ is plotted as Figure 3(b). One can clearly see that the anisotropy of the polarizability of the $\mathrm{C}_{18}$, and more specifically, the smaller the angle with the $\mathrm{C}_{18}$ ring plane, the greater the polarizability in the corresponding direction. The polarizability tensor reaches maximum magnitude in the $x-y$ plane, which is consistent with the polarizability component analysis above.
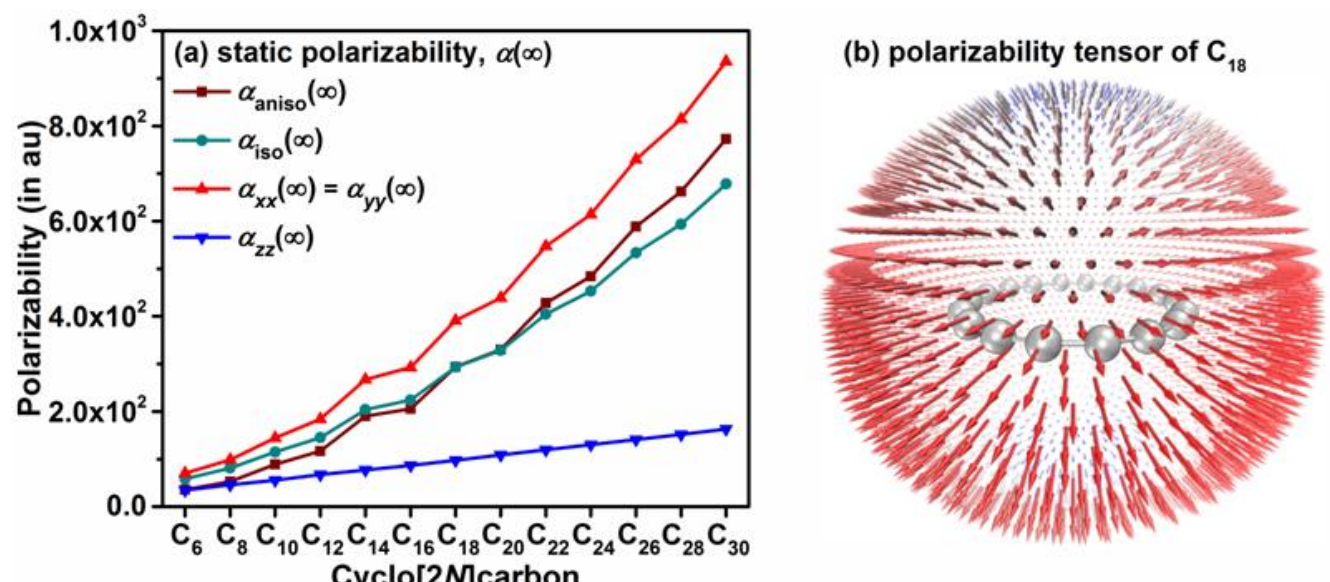

Figure 3. (a) Static polarizabilities $\alpha(\infty)$ of $\mathrm{C}_{2 N}$ systems and (b) unit sphere representation of static polarizability tensor of $\mathrm{C}_{18}$, the length and the colour of the arrows represent magnitude of polarizability in different directions. 
Figure 3(a) also reveals that with the enlargement of the ring size, the $\alpha_{\text {aniso }}(\infty)$, $\alpha_{\text {iso }}(\infty)$, as well as $\alpha_{x x}(\infty), \alpha_{y y}(\infty)$, and $\alpha_{z z}(\infty)$ all show different degrees of increase. The $\alpha_{z z}(\infty)$ rises slowly in a linear fashion, while the $\alpha_{x x}(\infty)$ and $\alpha_{y y}(\infty)$ increase rapidly and nonlinearly. This observation must derived from the fact that larger carbon ring possesses richer $\pi$ electrons and meantime provides broader region for electron displacement, which certainly result in stronger response of $\pi$ electrons to the external electric field along the ring plane. In order to extrapolate isotropic average polarizability to larger cyclocarbons, a second-order polynomial is fitted for $\alpha_{\text {iso }}(\infty)$ (Figure 4). The fitting quality is satisfactory $\left(R^{2}>0.998\right)$, therefore the equation fitted herein should be robust and useful for predicting $\alpha_{\text {iso }}(\infty)$ of carbon ring of arbitrarily large size.

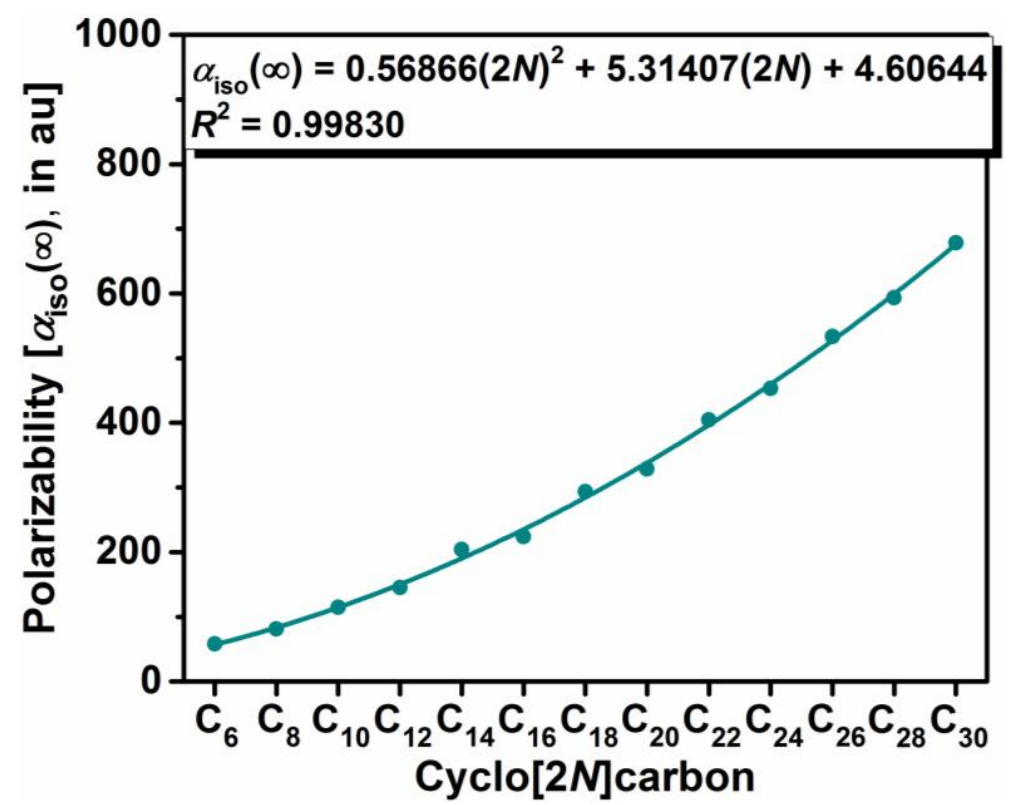

Figure 4. Curve-fitted isotropic average polarizability of the $\mathrm{C}_{2 N}$ systems with respect to atomic number.

Since all $\mathrm{C}_{2 N}$ rings studied in this work are nearly or completely centrosymmetric, their first-order hyperpolarizability must be negligible or exactly vanished ${ }^{45}$, hence this quantity is not included in our discussion. Even so, the $\mathrm{C}_{2 N}$ molecules are expected to be employed as an excellent $\pi$-linker in donor- $\pi$-acceptor type of organic dyes with good optical nonlinearity. 
Like the polarizability of the $\mathrm{C}_{2 N}$, the static second-order hyperpolarizability components parallel to the ring plane $\left[\gamma_{x x x x}(\infty)\right.$ and $\left.\gamma_{y y y y}(\infty)\right]$ are much larger than that in the perpendicular direction $\left[\gamma_{z z z z}(\infty)\right]$, and their difference becomes more and more prominent as the ring size increases [Figure 5(a)]. Specifically, the $\gamma_{z z z z}(\infty)$ rises very slowly with the increase of the ring size and its magnitude is always small. By contrast, the $\gamma_{x x x x}(\infty)$ and $\gamma_{y y y y}(\infty)$ increase sharply, especially for large cyclocarbons, and thus makes the average second-order hyperpolarizability, $\gamma_{\|}(\infty)$, display the similar variation trend. It is important to note that the variation of $\gamma_{\|}(\infty)$ is closely related to the parity of the number $N$ of the $\mathrm{C}_{2 N}$, that is, this quantity increases most significantly when $N$ is changed from even to odd, reflecting relatively better delocalization and thus stronger response capacity of the $\pi$ electrons in the systems satisfying Huckel's aromaticity rule, which again confirms the argument that macrocyclic aromaticity can be used as a fingerprint of molecular optical properties ${ }^{40}$.
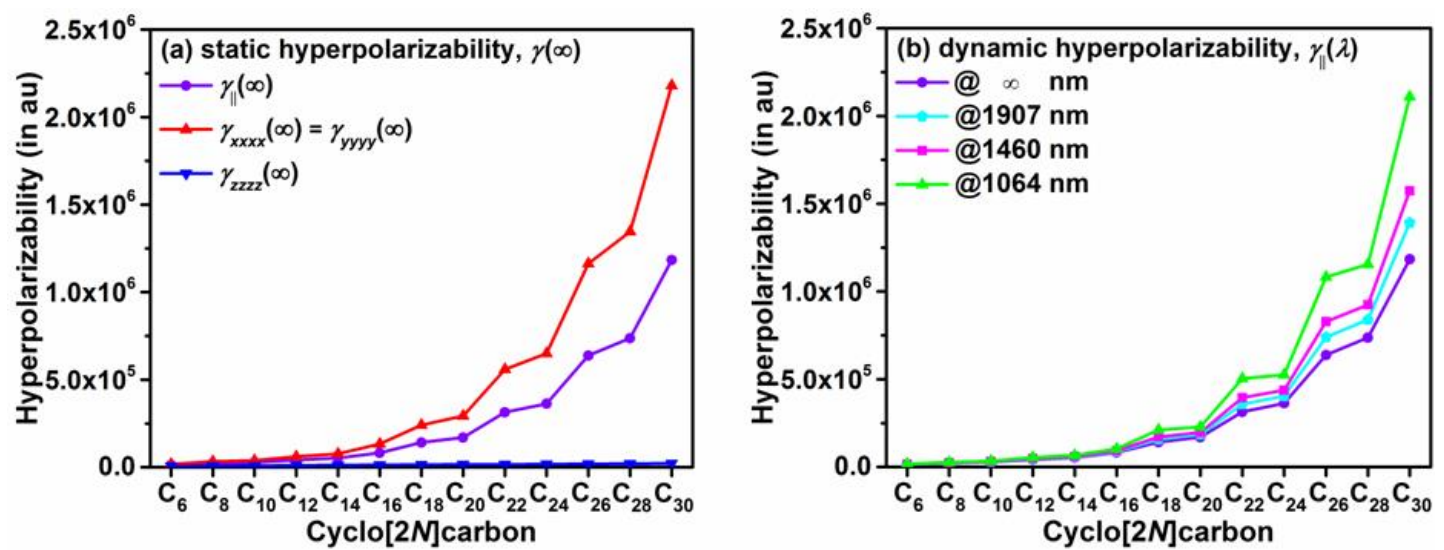

Figure 5. (a) static second-order hyperpolarizabilities $\gamma(\infty)$ of $\mathrm{C}_{2 N}$ systems and (b) frequency-dependent second-order hyperpolarizabilities $\gamma_{\|}(\lambda)$.

The frequency-dependent second-order hyperpolarizabilities $\left[\gamma_{\|}(\lambda), \lambda=1907\right.$, 1460, and $1064 \mathrm{~nm}]$ are substantially higher than the static one $\left[\gamma_{\|}(\infty)\right]$ for all $\mathrm{C}_{2 N}$ rings, and the resonance effect of the hyperpolarizability induced by incident light amplifies steadily with the light frequency [Figure 5(b)]. The variation behavior of $\gamma_{\|}(\lambda)$ with respect to the ring size is very similar to that of $\gamma_{\|}(\infty)$ described above. In order to build a general relationship between $\gamma_{\|}$and ring size, third-order polynomials 
are respectively fitted for $\gamma_{\|}(\infty)$ and $\gamma_{\|}(1064 \mathrm{~nm})$ (Figure 6$)$. The $\mathrm{C}_{2 N}$ rings with odd and even $N$ are considered separately due to their obviously different characters. The surprisingly satisfactory fitting quality $\left(R^{2}>0.999\right.$, see Table 1$)$ allows the fitted equations to be utilized for accurately predicting second-order hyperpolarizability for cyclocarbon of any size.

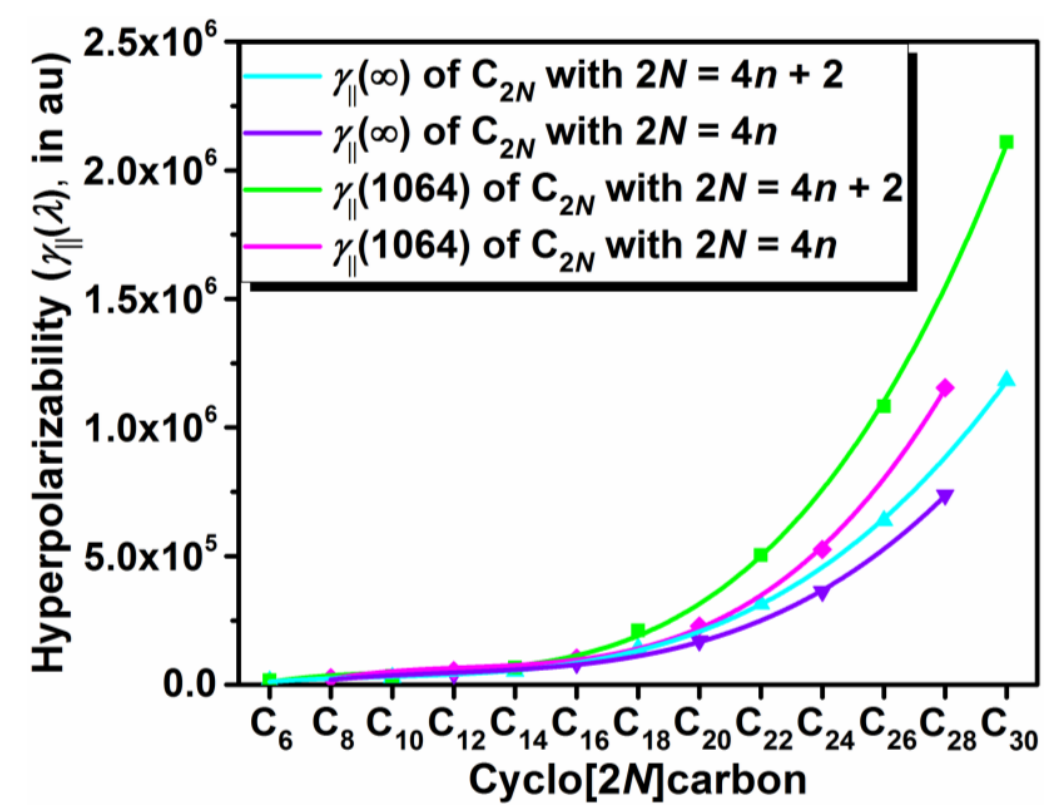

Figure 6. Curve-fitted average second-order hyperpolarizability of $\mathrm{C}_{2 N}$ systems in zero-frequency limit $(\lambda=\infty \mathrm{nm})$ and representative frequency-dependent fields $(\lambda=$ $1064 \mathrm{~nm}$ ) with respect to atomic number according to the parity of the $N$. 
Table 1. Fitting parameters related to curve-fitted average second-order hyperpolarizability in Figure 6

\begin{tabular}{|c|c|c|c|c|c|c|}
\hline \multirow{2}{*}{$\begin{array}{l}\text { field frequency } \\
\qquad(\lambda, \text { in } \mathrm{nm})\end{array}$} & \multirow{2}{*}{ atomic number } & \multicolumn{4}{|c|}{ polynomial coefficients in $\gamma_{\|}(\lambda)=a(2 N)^{3}+b(2 N)^{2}+c(2 N)+d$} & \multirow{2}{*}{$R^{2}$} \\
\hline & & $a$ & $b$ & $c$ & $d$ & \\
\hline & $2 N=4 n+2$ & 129.41145 & -3772.90320 & 40083.87279 & -120839.88992 & 0.99985 \\
\hline & & 141.41679 & -4979.65562 & 63438.86356 & -241406.85312 & 0.99977 \\
\hline \multirow{2}{*}{1064} & $2 N=4 n+2$ & 263.82812 & -8235.99454 & 89212.14252 & -285581.60372 & 0.99968 \\
\hline & $2 N=4 n$ & 266.91836 & -9950.29882 & 128474.63700 & -504640.47133 & 0.99960 \\
\hline
\end{tabular}




\section{Conclusions}

In summary, we have made theoretical predictions and analyses on the geometry, electronic structure, photophysical property, and optical nonlinearity of the intriguing $\mathrm{C}_{2 N}$ allotropes with $N=3-15$. As the number of ring members increases, their maximum absorption wavelength of electronic spectra gradually red-shifts and the absorption intensity increases steadily. Meanwhile, the molecular (hyper)polarizability increases significantly irrespective of the frequency of incident light. Variation behaviors of these properties are found to be quite regular and closely dependent of the parity of $N$, implying the essential relevance of optical properties to molecular aromaticity. The optical properties of larger cyclocarbons beyond the computational power can be reliably estimated by extrapolating according to the fitting equations provided in this work. Considering that the cyclocarbons have strong absorption in the ultraviolet region, they are expected to be utilized as ultraviolet filter materials with excellent performance and, more important, the filtering range can be tunable according to actual demand by controlling the size of the carbon ring. In view of the considerable anisotropy of the (hyper)polarizability, the carbocyclic crystals have potential to become nonlinear optical materials with excellent anisotropy characteristics.

\section{Notes}

The authors declare no competing financial interest.

\section{Acknowledgments}

This work was partially supported by The Natural Science Foundation of the Jiangsu Higher Education Institutions of China (Grant No. 18KJA180005).

\section{Supporting Information Available}

Optimized Cartesian coordinates; average atomization energy; density-of-states map and molecular orbital degeneracy; designation of the molecular orbital type; curvefitted frontier molecular orbital energy levels; hole and electron distributions; $\mathrm{X}$ and $\mathrm{Y}$ components of transition dipole moment density; polarizability and second-order hyperpolarizability. 


\section{References}

(1) Kaiser K, Scriven LM, Schulz F, Gawel P, Gross L, Anderson HL. An sphybridized molecular carbon allotrope, cyclo[18]carbon. Science 2019; 365(6459): 1299-1301.

(2) Kozuch S, Nandi A, Solel E. Carbon tunneling in the automerization of cyclo[18]carbon. Chem Eur J 2020; 26(3): 564-753.

(3) Stasyuk AJ, Stasyuk OA, Solà M, Voityuk AA. Cyclo[18]carbon: The smallest all-carbon electron acceptor. Chem Commun 2020; 56(3): 352-355.

(4) Zhang L, Li H, Feng YP, Shen L. Diverse transport behaviors in cyclo[18]carbonbased molecular devices. J Phys Chem Lett 2020; 11(7): 2611-2617.

(5) Pereira ZS, da Silva EZ. Spontaneous symmetry breaking in cyclo[18]carbon. J Phys Chem A 2020; 124(6): 1152-1157.

(6) Baryshnikov GV, Valiev RR, Kuklin AV, Sundholm D, Ågren H. Cyclo[18]carbon: Insight into electronic structure, aromaticity and surface coupling. J Phys Chem Lett 2019; 10(21): 6701-6705.

(7) Haley MM. Cyclo[18]carbon, the newest member of the family of carbon allotropes. Chem 2019; 5(10): 2517-2519.

(8) Rahman MZ, Edvinsson T. Rational design and resolution of the mystery of the structure of cyclo[18]carbon. J Mater Chem A 2020; 8(17): 8234-8237.

(9) Fedik N, Kulichenko M, Steglenko D, Boldyrev AI. Can aromaticity be a kinetic trap? Example of mechanically interlocked aromatic [2-5]catenanes built from cyclo[18]carbon. Chem Commun 2020; 56(18): 2711-2714.

(10) Hussain S, Chen H, Zhang Z, Zheng H. Vibrational spectra and chemical imaging of cyclo[18]carbon by tip enhanced Raman spectroscopy. Chem Commun 2020; 56(15): 2336-2339.

(11) Pichierri F. Boron-nitrogen analogues of cyclo[18]carbon. Chem Phys Lett 2020; 738: 136860.

(12) Jiang Y, Mattioli EJ, Calvaresi M, Wang Z. Theoretical design of an ultrafast supramolecular rotor composed of carbon nano-rings. Chem Commun 2020; 56(79): $11835-11838$.

(13) Charistos ND, Muñoz-Castro A. Induced magnetic field in sp-hybridized carbon rings: Analysis of double aromaticity and antiaromaticity in cyclo[2N]carbon allotropes. Phys Chem Chem Phys 2020; 22(17): 9240-9249. 
(14) Qin B, Zhang Q, Li Y, Yang G, Yu H, Peng F. Mechanistic insights into the electrochemical reduction of $\mathrm{CO}_{2}$ on cyclo[18]carbon using density functional theory calculations. ChemElectroChem 2020; 7(8): 1838-1842.

(15) Li M, Gao Z, Han Y, Zhao Y, Yuan K, Nagase S, Ehara M, Zhao X. Potential molecular semiconductor devices: Cyclo- $\mathrm{C}_{n}(n=10$ and 14) with higher stabilities and aromaticities than acknowledged cyclo- $\mathrm{C}_{18}$. Phys Chem Chem Phys 2020; 22(8): 4823-4831.

(16) Raeber AE, Mazziotti DA. Non-equilibrium steady state conductivity in cyclo[18]carbon and its boron nitride analogue. Phys Chem Chem Phys 2020; 22(41): 23998-24003.

(17) Fang S, Hu Y. Cyclo[18]carbon as an ultra-elastic molecular O-ring with unique mechanical properties. Carbon 2021; 171: 96-103.

(18) Dai C, Chen D, Zhu J. Achieving adaptive aromaticity in cyclo[10]carbon by screening cyclo[n]carbon ( $\mathrm{n}=8-24)$. Chem Asian J 2020; 15(14): 2187-2191.

(19) Liu Z, Lu T, Chen Q. An sp-hybridized all-carboatomic ring, cyclo[18]carbon: Bonding character, electron delocalization, and aromaticity. Carbon 2020; 165: $468-475$

(20) Liu Z, Lu T, Chen Q. An sp-hybridized all-carboatomic ring, cyclo[18]carbon: Electronic structure, electronic spectrum, and optical nonlinearity. Carbon 2020; 165: 461-467.

(21) Liu $\mathrm{Z}, \mathrm{Lu} \mathrm{T}$, Chen Q. Intermolecular interaction characteristics of the allcarboatomic ring, cyclo[18]carbon: Focusing on molecular adsorption and stacking. Carbon 2021; 171: 514-523.

(22) Liu Z, Lu T, Chen Q. Vibrational spectra and molecular vibrational behaviors of all-carboatomic rings, cyclo[18]carbon and its analogues. Chem Asian J 2021; 16: $56-63$.

(23) $\mathrm{Lu} \mathrm{T}$, Chen Q. Ultrastrong regulation effect of electric field on all-carboatomic ring, cyclo[18]carbon. ChemPhysChem 2021; 22: 386-395.

(24) Scriven LM, Kaiser K, Schulz F, Sterling AJ, Woltering SL, Gawel P, Christensen KE, Anderson HL, Gross L. Synthesis of cyclo[18]carbon via debromination of $\mathrm{C}_{18} \mathrm{Br}_{6}$. J Am Chem Soc 2020; 142(30): 12921-12924.

(25) Chai J-D, Head-Gordon M. Long-range corrected hybrid density functionals with damped atom-atom dispersion corrections. Phys Chem Chem Phys 2008; 10(44): $6615-6620$. 
(26) Weigend F, Ahlrichs R. Balanced basis sets of split valence, triple zeta valence and quadruple zeta valence quality for $\mathrm{H}$ to $\mathrm{Rn}$ : Design and assessment of accuracy. Phys Chem Chem Phys 2005; 7(18): 3297-3305.

(27) Mardirossian N, Head-Gordon M. $\omega$ B97M-V: A combinatorially optimized, range-separated hybrid, meta-GGA density functional with VV10 nonlocal correlation. J Chem Phys 2016; 144(21), 214110.

(28) Jensen F. Introduction to computational chemistry. John Wiley \& Sons, West Sussex, 2017.

(29) Yanai T, Tew D, Handy N. A new hybrid exchange-correlation functional using the coulomb-attenuating method (CAM-B3LYP). Chem Phys Lett 2004; 393(1-3): $51-57$.

(30) Dunning TH, Jr. Gaussian basis sets for use in correlated molecular calculations.

I. The atoms boron through neon and hydrogen. J Chem Phys 1989; 90(2): 10071023.

(31) Baranowska A, Sadlej AJ. Polarized basis sets for accurate calculations of static and dynamic electric properties of molecules. J Comput Chem 2010; 31(3): 552560.

(32) Buckingham AD. Permanent and induced molecular moments and mong-range intermolecular forces. Adv Chem Phys 1967; 12: 107-142.

(33) Mclean AD, Yoshimine M. Theory of molecular polarizabilities. J Chem Phys 1967; 47(6): 1927-1935.

(34) Willetts A, Rice JE, Burland DM, Shelton DP. Problems in the comparison of theoretical and experimental hyperpolarizabilities. J Chem Phys 1992; 97(10): 7590-7599.

(35) Frisch MJ, Trucks GW, Schlegel HB, Scuseria GE, Robb MA, Cheeseman JR, et al. Gaussian 16, revision A.03; Gaussian, Inc.: Wallingford, CT, 2016.

(36) Neese F. Software update: the ORCA program system, version 4.0, WIREs Comput Mol Sci 2018; 8(1): e1327.

(37) Lu T, Chen F. Multiwfn: A multifunctional wavefunction analyzer. J Comput Chem 2012; 33(5): 580-592.

(38) Humphrey W, Dalke A, Schulten K. VMD: Visual molecular dynamics. J Mol Graphics 1996; 14(1): 33-38. 
(39) Zade SS, Zamoshchik N, Bendikov M. From short conjugated oligomers to conjugated polymers. Lessons from studies on long conjugated cligomers. Accounts Chem Res 2011; 44(1): 14-24.

(40) Woller T, Geerlings P, De Proft F, Champagne B, Alonso M. Fingerprint of aromaticity and molecular topology on the photophysical properties of octaphyrins. J Phys Chem C 2019; 123(12): 7318-7335.

(41) Liu Z, Lu T, Hua S, Yu Y. Aromaticity of Hückel and Möbius topologies involved in conformation conversion of macrocyclic [32] octaphyrin(1.0.1.0.1.0.1.0): Refined evidence from multiple visual criteria. J Phys Chem C 2019; 123(30): 18593-18599.

(42) Liu Z, Lu T. Controllable photophysical and nonlinear properties in conformation isomerization of macrocyclic [32] octaphyrin(1.0.1.0.1.0.1.0) involving Hückel and Möbius topologies. J Phys Chem C 2020; 124(1): 845-853.

(43) Lu T. Multiwfn manual, version 3.7, Section 3.21.1, available at http://sobereva.com/multiwfn/ (accessed on Oct 1, 2020).

(44) Tuer A, Krouglov S, Cisek R, Tokarz D, Barzda V. Three-dimensional visualization of the first hyperpolarizability tensor. J Comput Chem 2011; 32(6): $1128-1134$.

(45) Günter P, Nonlinear optical effects and materials, Springer-Verlag, Berlin, 2000. 